\title{
Regional Flow and Vertical Heat Transport in Groundwater. Numerical Solution for the Study of Temperature Profiles
}

\author{
Iván Alhama ${ }^{1}$, Gonzalo García-Ros ${ }^{1}$, José Antonio Jiménez-Valera1 \\ ${ }^{1}$ Technical University of Cartagena \\ Paseo Alfonso XIII, 52, Cartagena, Spain \\ ivan.alhama@upct.es; gonzalo.garcia@upct.es; joseantoniojimenez1996@hotmail.com
}

\begin{abstract}
In this work, temperature patterns and profiles have been obtained in large 2-D groundwater scenarios, with constant and horizontal regional flow and thermal conditions that reproduce approximately real cases, such as the daily or seasonal variation of the soil surface temperature. For this purpose, a numerical model based on the network simulation method has been designed and applied to real scenarios to determine the correlation between derived temperature profiles and groundwater flow. The results of this first work allow to see more closely the possibility of addressing a much more complex problem, such as the determination of the regional velocity field from temperature profiles read from in situ wells.
\end{abstract}

Keywords: Porous media; groundwater flow; heat transport; temperature profiles.

\section{Introduction}

The coupled problems of fluid flow and heat transport in porous media, particularly those related to underground hydrology, have aroused great interest in recent times for their application in the field of geothermal, i.e. the energy use of thermal sources in the subsoil $[1,2]$. Their study has even led to the establishment by the scientific community of standard or benchmark problems that allow verifying numerical codes in this field since, in general, they have no analytical solution. Among these problems are those of Bénard, Yusa and Elder [3], which have given rise to numerous scientific publications. In all of them, temperature patterns or profiles are coupled to the velocity field, mainly because the flow equation contains a density-driven term.

However, when the temperature range of the problem is narrow and the velocity field is essentially imposed by an external pressure gradient or piezometric levels (with negligible influence of flotation) that would cause a regional flow, the problem, although simpler in its mathematical model, can be potentially very useful. The straight profile of temperatures that would occur in the absence of regional flow under stationary conditions, away from the water flow inlet boundary (resulting in a constant heat flow to the bottom), would be distorted and curved by the effect of the horizontal drag when there is regional flow. Thus, at all times, the temperature profile with depth is determined by the thermal properties of the ground and the value of the regional flow, apart from the influence of the thermal boundary conditions of the scenario. If, as it actually happens, the outside temperature is a seasonal function of time, its profile in the vicinity of the ground surface changes continuously, but its experimental measurement could allow the calculation of regional water flow. This is the objective of the present communication, to determine the connection between the underground water flow and the form of the temperature profiles that can be measured in a well. This would allow us to consider in the future the determination of the regional velocity field from the reading (direct and hardly expensive) of temperature profiles in wells.

The first approach to this problem consists in investigating the shape of the temperature profiles in a 2-D scenario of sufficient extension, with constant regional flow in the horizontal direction and thermal conditions that reproduce approximately real cases, such as harmonic variation of the soil surface temperature and constant temperature at the bottom of the domain.

For this study, a numerical model has been designed based on the network simulation method, whose reliability has been verified in numerous engineering applications $[4,5]$. The elaboration of the model is briefly introduced and it is applied to real scenarios to determine the characteristics of the correlation between the profiles obtained and the regional flows that determine them. 


\section{The physical and mathematical models}

Figure 1 is a physical scheme of the scenario. As for the mechanical problem, a regional water flow induces a constant velocity field $\left(\mathrm{v}_{\mathrm{o}}\right)$ throughout the domain. As for the thermal problem (which assumes the phenomena of diffusion and convection in the porous medium), the following boundary conditions are imposed: i) constant temperature of the inlet fluid (first class or Dirichlet condition at the left vertical boundary, $x=0$ ), ii) seasonally time-dependent temperature at the upper horizontal boundary (special first class condition at $\mathrm{y}=\mathrm{H}$ ), iii) zero heat flow at the bottom (second class or Neumann homogeneous condition at $\mathrm{y}=0$ ), and iv) constant temperature condition at the flow outlet front, $\mathrm{x}=\mathrm{L}$. In order to eliminate the influence of the thermal flow output condition on the temperature profile, a sufficiently long horizontal domain has been adopted, reading these profiles in the previous region to the influence of this condition. The same precaution is taken regarding the influence of the fluid inlet temperature on the profile. These regions, before and after the profile measurement, depend on both the regional velocity and the thermal fluid properties.

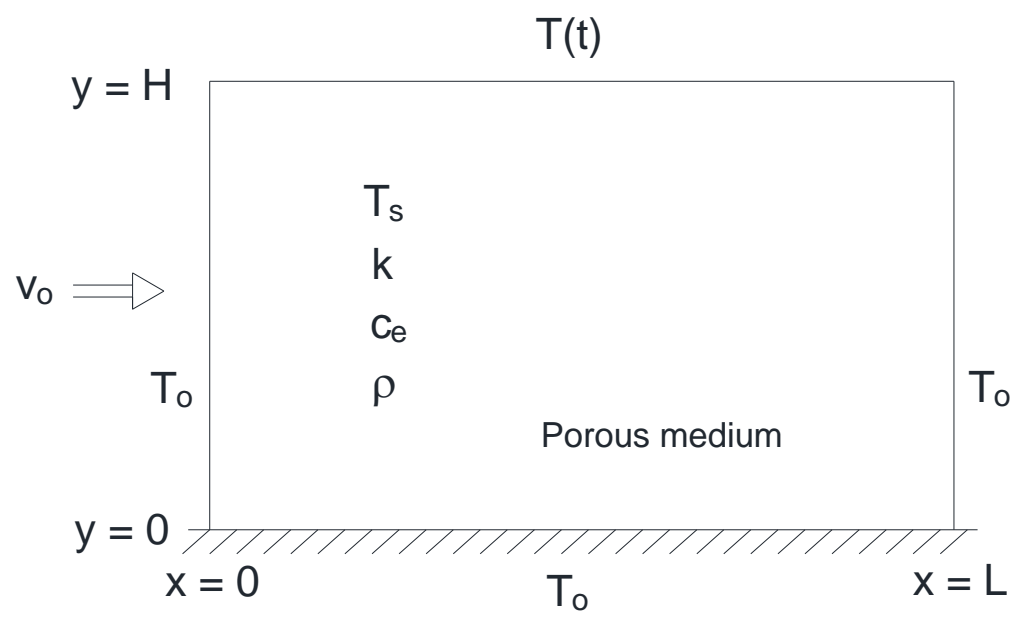

Fig. 1: Physical scheme of the problem.

The mathematical 2-D model is formed by the following set of equations:

$$
\begin{gathered}
\left(\rho c_{e}\right) \frac{\partial \mathrm{T}}{\partial \mathrm{t}}-\mathrm{k} \nabla^{2}(\mathrm{~T})+\left(\rho \mathrm{c}_{\mathrm{e}} \mathrm{v}_{\mathrm{o}} \frac{\partial \mathrm{T}}{\partial \mathrm{x}}\right)=0 \\
\mathrm{~T}_{(\mathrm{x}=0, \mathrm{y}, \mathrm{t})}=\mathrm{T}_{(\mathrm{x}=\mathrm{L}, \mathrm{y}, \mathrm{t})}=\mathrm{T}_{\mathrm{o}} \\
\mathrm{T}_{(\mathrm{x}, \mathrm{y}=0, \mathrm{t})}=\mathrm{T}_{\mathrm{o}} \\
\mathrm{T}_{(\mathrm{x}, \mathrm{y}=\mathrm{H}, \mathrm{t})}=\mathrm{T}_{\mathrm{m}} \operatorname{sen}\left(\omega_{\mathrm{o}} \mathrm{t}\right) \\
\mathrm{T}_{(\mathrm{x}, \mathrm{y}, \mathrm{t}=0)}=\mathrm{T}_{\mathrm{s}} \\
\mathrm{v}(\mathrm{x}, \mathrm{y}, \mathrm{t})=\mathrm{v}_{\mathrm{o}}
\end{gathered}
$$

Eq. (1) is that of heat transfer in the soil, where $\mathrm{k}$ is the thermal conductivity of the soil $\left(\mathrm{Jm}^{-1} \mathrm{~s}^{-1} \mathrm{~K}^{-1}\right), \rho$ the density $\left(\mathrm{kg} / \mathrm{m}^{3}\right)$ and $\mathrm{c}_{\mathrm{e}}$ the specific heat $\left(\mathrm{Jm}^{-3} \mathrm{~K}^{-1}\right)$. There is a diffusion in both spatial directions, a drag or convection in the OX direction and a local storage, quantities that are balanced in each volume or cell element. Temperature (T) is the dependent variable while spatial coordinates $(x, y)$ and time $(t)$ are the independent ones. Eq. (2) represents the isothermal boundary conditions at the vertical sides $\mathrm{x}=0$ and $\mathrm{x}=\mathrm{L}$ of the domain, as well as eq. (3) at the bottom, and eq. (4) can be, for example, the condition at the upper surface. $\mathrm{T}_{(\mathrm{x}, \mathrm{y}=\mathrm{H}, \mathrm{t})}$ is an harmonic time-dependent temperature that approaches the seasonal variations of the environment with $T_{m}$ the mean temperature, $\omega_{o}=\frac{2 \pi}{t_{o}}$ the pulsation and $t_{0}$ the time period of 
the oscillation. Eq. (5) is the initial temperature of the soil and eq. (6) the solution of the flow velocity field at the domain. This is the model studied in this work although it is planned to extend it to more complex scenarios in future works. For example, velocity field dependent on depth, water table at levels below the soil surface, scenarios with sloping bottoms that that imply the existence of vertical velocity components, convection and radiation incident fluxes on the soil surface, etc. As etc. As we will see below, from the point of view of the network model design, these new scenarios do not entail special restrictions since the network method [6] has successfully addressed problems of similar complexity [4, 5].

\section{The network model}

This is designed based on the finite-difference differential equation that derives from the spatial discretization of governing eq. (1), retaining time as a continuous variable. For a volume element of an isotropic and homogeneous soil, this equation writes as

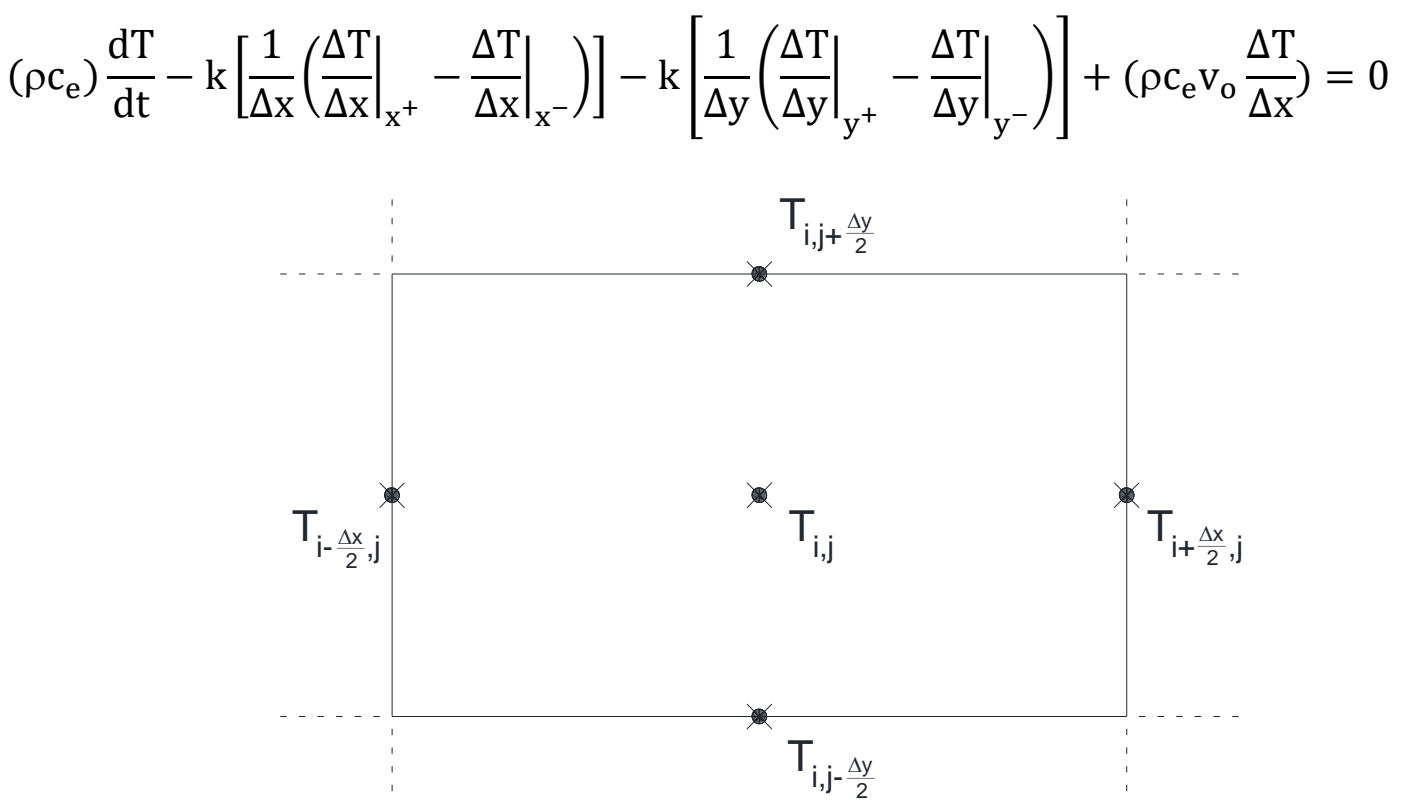

Fig. 2: Nomenclature of temperatures in the volume element.

Now, we set the analogy between physical quantities 'electric current $\sim$ heat flux' and 'electric potential $\sim$ temperature'. Using the nomenclature of Figure 2 for the volume element or cell, each addend of the former equation is assumed to be an electric current through a branch of the network (or electric circuit) that balances with the currents of the other addends in their respective branches in a common node. The voltage in this node, once the balance among currents is satisfied, is the temperature of the cell. The constitutive equation between current and voltage through the electric component implemented in each branch must fit the mathematical expression of the related addend. Defining the soil thermal diffusivity as $D=\frac{k}{\rho c_{e}}$ $\left(\mathrm{m}^{2} \mathrm{~s}^{-1}\right)$ and re-organizing the above eq. (7), we have

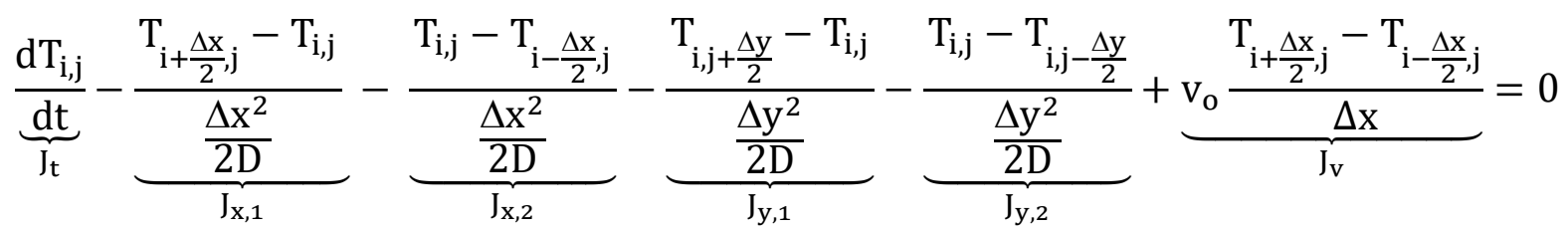


The current $J_{t}$ is implemented by a capacitor of value $C=1$ since its constitutive equation is $J_{C}=C \frac{d V_{C}}{d t}$ while the terms $J_{x, 1}, J_{x, 2}, J_{y, 1}$ and $J_{y, 2}$ do it with resistors of value $R_{x, 1}=R_{x, 2}=\frac{\Delta x^{2}}{2 D}, R_{y, 1}=R_{y, 2}=\frac{\Delta y^{2}}{2 D}$ since the constitutive equation of such passive element is given by $J_{R}=\frac{V_{R}}{R}$. Finally, $J_{v}$ is implemented by a voltage-controlled current source (G), an active element of the circuit whose output, programmed by software, is given by the mathematical expression of $\mathbf{J}_{\mathrm{v}}$. The network of the cell, shown in Figure 3, extends to the entire domain through simple ideal contacts between adjacent cells. Moreover, boundary conditions have to be added. Thus, vertical sides, eq. (2), are implemented by constant voltage sources, as well as eq. (3) in the bottom side, where in the upper side, eq. (4), a time-dependent voltage source is needed. Finally, initial condition given by eq. (5) is implemented by charging the capacitors with an initial voltage of value $\mathrm{T}_{\mathrm{s}}$.

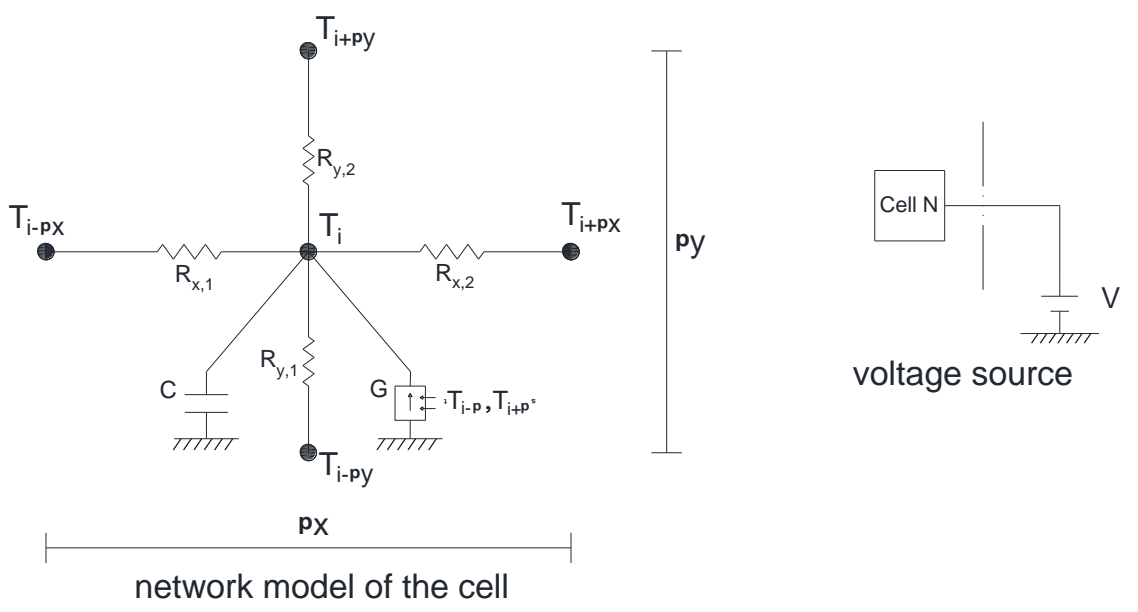

Fig. 3: Network model of the cell (left) and voltage boundary condition (right).

Once the model is introduced in a circuit simulation computer code (the free code Ngspice [7] is the one chosen in this work) it is run, giving the time-dependent solutions of the voltage (temperature) at all nodes and the currents (heat flux) at all branches. Thanks to the powerful computing algorithms implemented in these codes, the simulation provides a practically exact solution of the model, relegating errors to the mesh size chosen. For meshes above 50x50 cells, computation times are low (5-30 s) and errors fall below 1\% [8], a generally accepted value in engineering.

\section{Applications}

Two different scenarios have been chosen. The first one has the following data:

$\mathrm{L}=40 \mathrm{~m}, \mathrm{H}=10 \mathrm{~m}, \mathrm{v}_{\mathrm{o}}=0.2 \mathrm{~ms}^{-1}, \mathrm{k}=1 \mathrm{Jm}^{-1} \mathrm{~s}^{-1} \mathrm{~K}^{-1}, \rho c_{\mathrm{e}}=2.7 \times 10^{4} \mathrm{Jm}^{-3} \mathrm{~K}^{-1}, \mathrm{D}=2.7 \times 10^{-4} \mathrm{~m}^{2} \mathrm{~s}^{-1}$

$\mathrm{T}_{\mathrm{o}}=0 \mathrm{~K}, \mathrm{~T}(\mathrm{t})=1 \mathrm{~K}, \mathrm{~T}_{\mathrm{s}}=0 \mathrm{~K}$

$\mathrm{N}_{\mathrm{x}}=80, \Delta \mathrm{x}=40 / 80=0.5 \mathrm{~m}, \mathrm{~N}_{\mathrm{y}}=20, \Delta \mathrm{y}=10 / 20=0.5 \mathrm{~m}$, Simulation time $=1000$ hours.

The simulation results, with a calculation time of around $20 \mathrm{~s}$, are shown below. Thus, Figure 4 shows the temperature patterns for different simulation times. At 50 hours it can be seen that after about 20 meters from the left border, where the water flow comes from, a constant temperature profile is achieved, independent on the OX axis position. However, this is not a stationary situation, as we can see that at 100 hours the profile has evolved in depth (in this case the profile is more or less constant after 30 meters on the OX axis). It is around 500 hours when the stationary temperature profile is reached, since the thermal gradient is the same throughout the medium. However, the distance on the OX axis to reach the stationary situation rises to 100 meters (Figure 5) for which it has been necessary to extend the length $\mathrm{L}$ of the problem to 120 meters. 

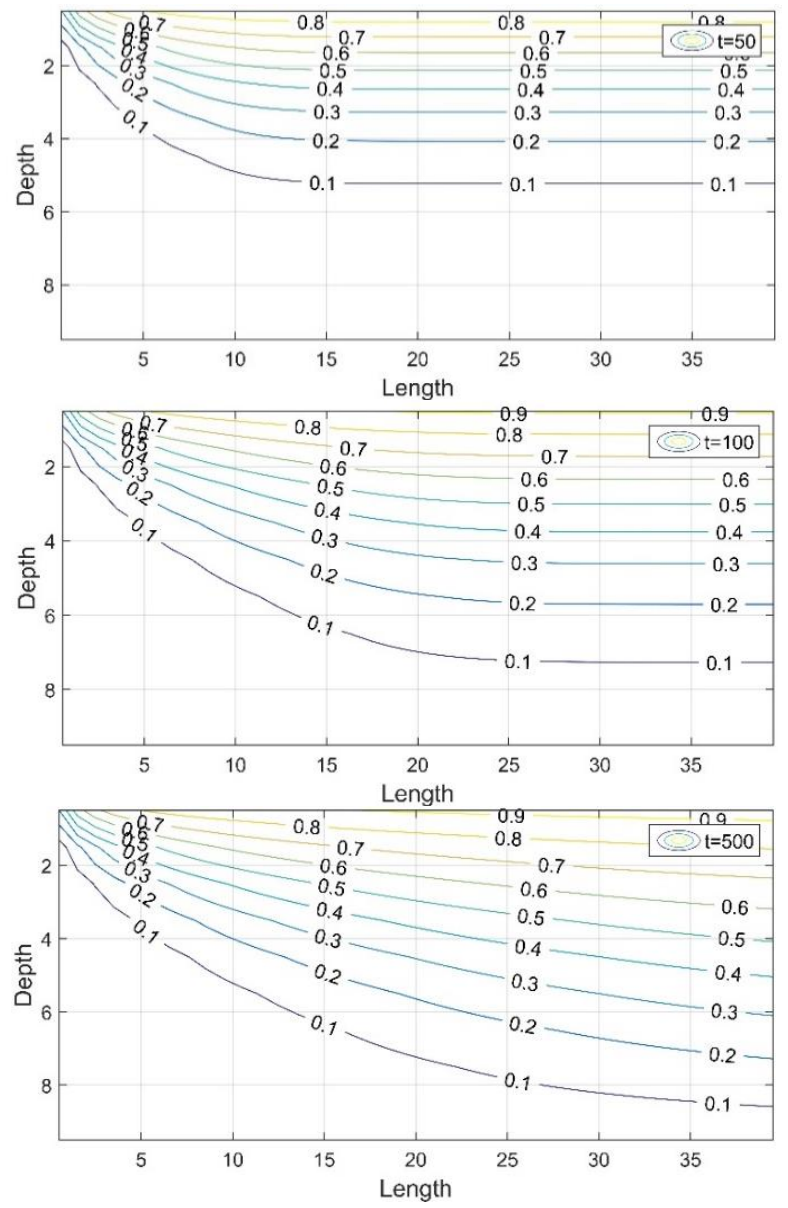

Fig. 4: Temperature patterns of the first scenario chosen (H=10m, $\mathrm{L}=40 \mathrm{~m}, \mathrm{t}=50,100,500$ hours).
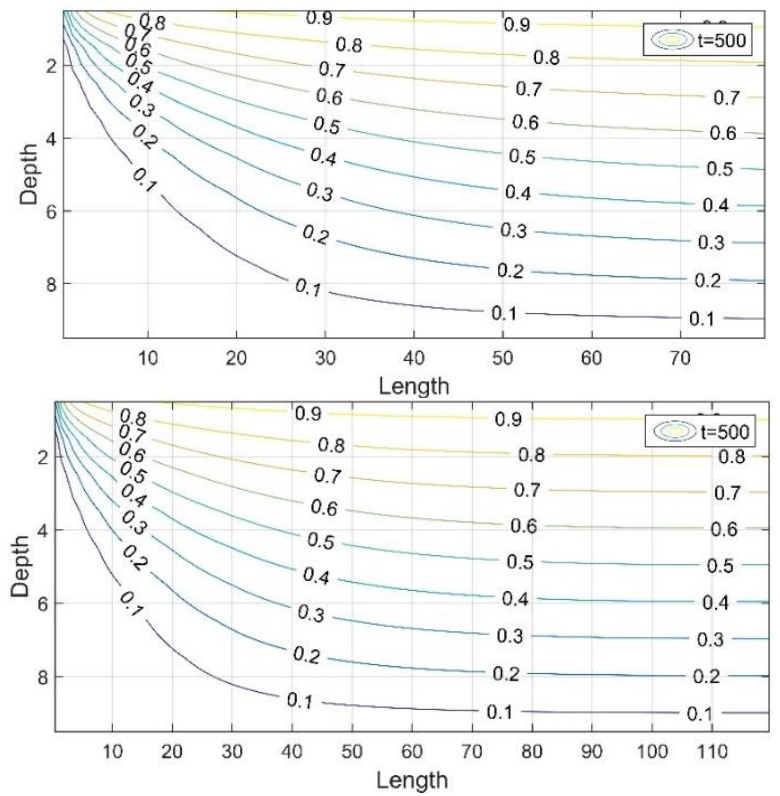

Fig. 5: Temperature patterns of the first scenario chosen ( $\mathrm{H}=10 \mathrm{~m}, \mathrm{~L}=80,120 \mathrm{~m}, \mathrm{t}=500$ hours). 
In view of these data it is observed how the temperature profiles, at times long enough for the stationary situation to be reached, are initially dependent on the position and then become independent in the central region. Figure 6 shows the different depth-temperature profiles obtained in the previous scenario, for different OX distances (5, 40, 80 and 120 meters) and for different times since the start of the problem (50,100 and 500 hours). These last profiles ( $\mathrm{t}=500$ hours) are the ones that would be expected to be registered if in a field study we measure the evolution of the temperature with the depth in a borehole or well located in the considered OX position.

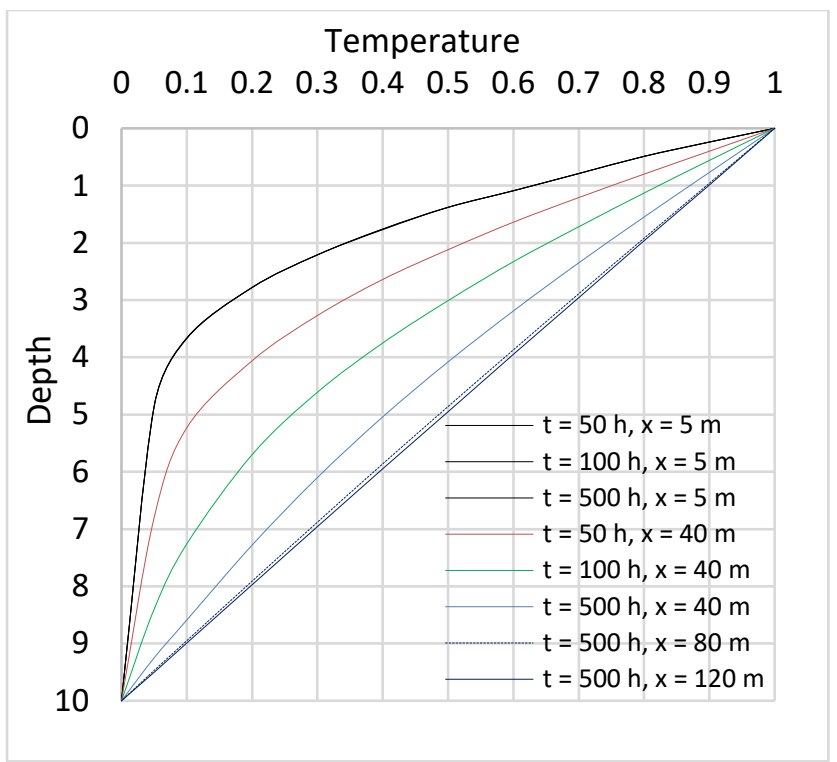

Fig. 6: Temperature profiles of the first scenario chosen.

The second scenario differs from the first in the following data:

$\mathrm{L}=120 \mathrm{~m}, \mathrm{~T}(\mathrm{t})$ given in Figure 7, $\mathrm{N}_{\mathrm{x}}=120, \Delta \mathrm{x}=120 / 120=1 \mathrm{~m}$.

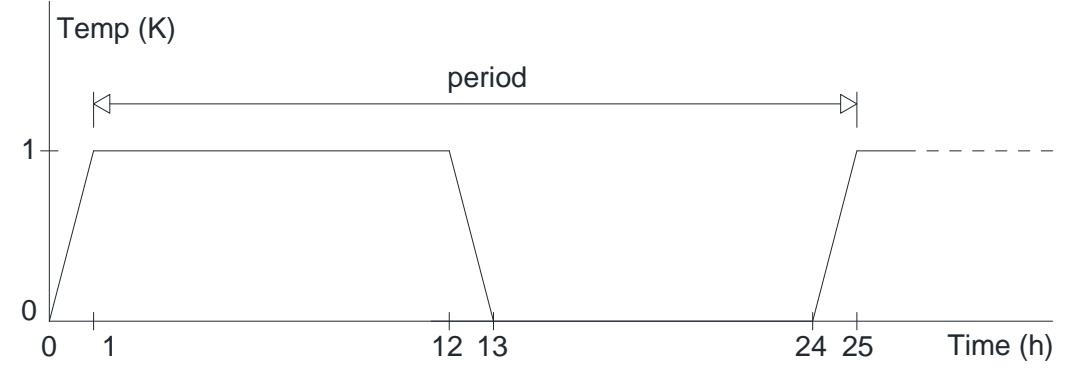

Fig. 7: Periodic function of the temperature in the soil surface.

For this second scenario, the stationary situation is never reached, since the temperature varies periodically in the upper boundary. However, what does happen is that after approximately 600 hours have elapsed, the temperature patterns and profiles begin to repeat continually, as long as we compare them at the same time in the cycle or period. Thus, in Figures 8 and 9 the depth-temperature patterns and profiles are represented for the moments immediately before the beginning of the change of the temperature in the upper side (multiples of hours 12 and 24 hours, endings of $\mathrm{T}=0$ and $\mathrm{T}=1$, respectively). 

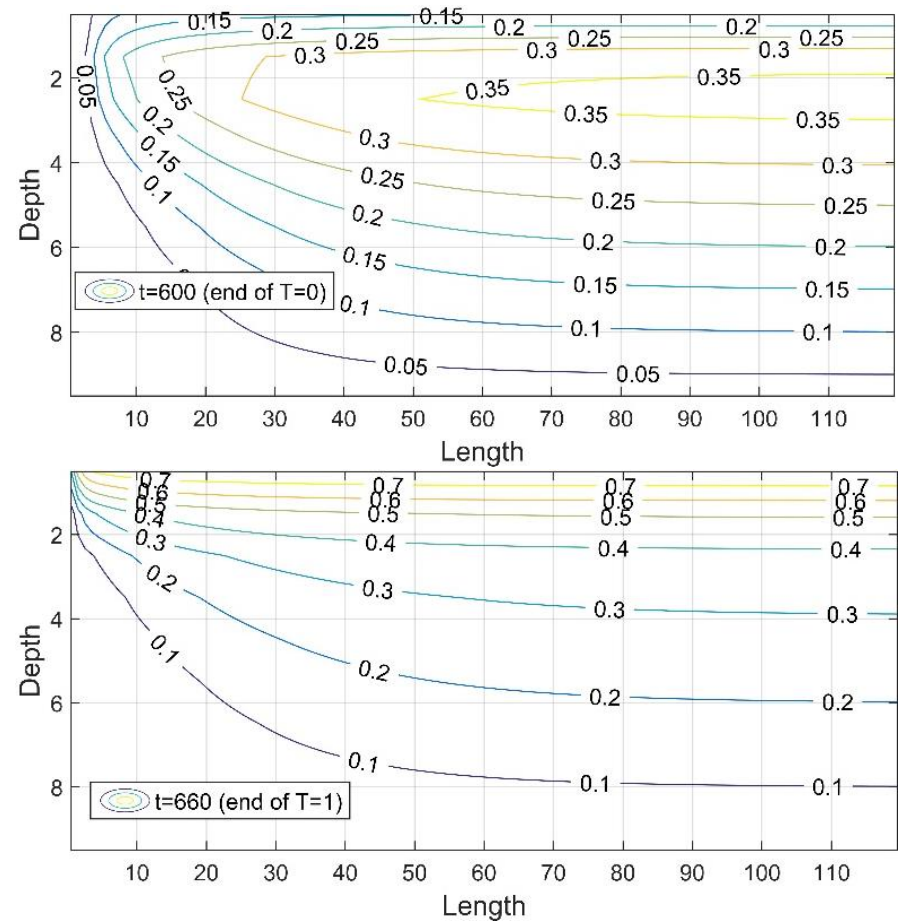

Fig. 8: Temperature patterns of the second scenario chosen ( $\mathrm{H}=10 \mathrm{~m}, \mathrm{~L}=120 \mathrm{~m}, \mathrm{t}=600,660$ hours).

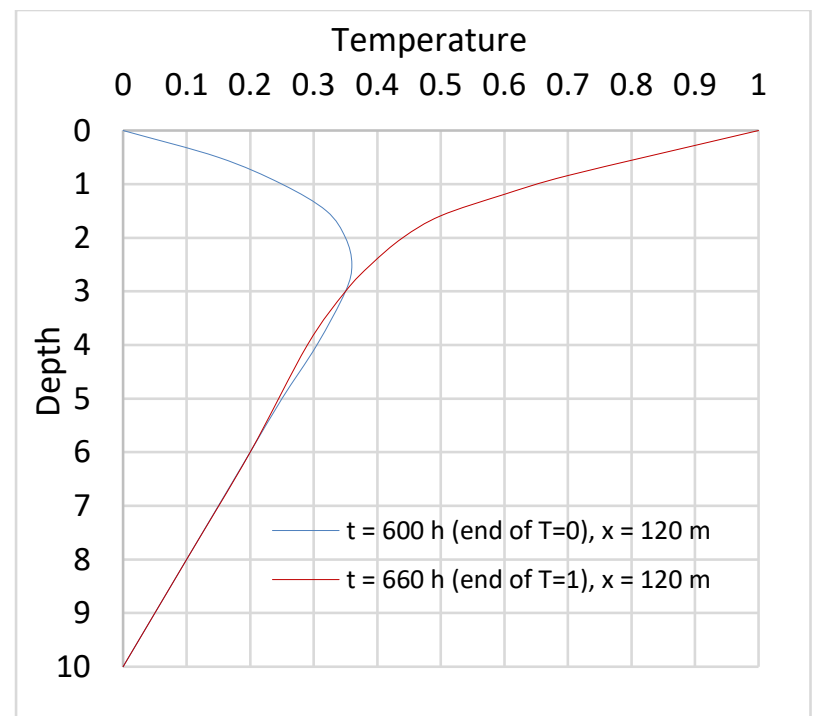

Fig. 9: Temperature profiles of the second scenario chosen.

\section{Conclusion}

The network method has been successfully applied to different scenarios of heat transport within a porous medium through which underground water flows. Through this technique, different temperature boundary conditions have been implemented, so that simulated scenarios are very close to the reality observed in this type of problems. 
The tendencies observed in the temperature patterns and profiles have provided very valuable information, since the lengths and the times in which the stationary, or quasi-stationary, state is reached have been determined.

The results obtained bring us closer to a horizon in which groundwater flux could be obtained from real field temperature readings.

\section{Acknowledgements}

We would like to thank the SéNeCa Foundation for the scholarship awarded to José Antonio Jiménez Valera, which will allow us to continue this investigation.

\section{References}

[1] E. Di Sipio et al., "Rock thermal conductivity as key parameter for geothermal numerical models," Energy Procedia, vol. 40, pp. 87-94, 2013.

[2] M. Barla, A. D. Donna, and M. Baralis, "City-scale analysis of subsoil thermal conditions due to geothermal exploitation," Environmental Geotechnics, pp. 1-11, 2018.

[3] M. Cánovas, Caracterización adimensional y simulación numérica de escenarios patrón (Bénard, Yusa y Elder) de procesos geotérmicos de flujo y transporte. Doctoral dissertation. Universidad Politécnica de Cartagena, 2014.

[4] G. García-Ros, I. Alhama and J. L. Morales, "Numerical simulation of nonlinear consolidation problems by models based on the network method," Applied Mathematical Modelling, vol. 69, pp. 604-620, 2019.

[5] M. Cánovas et al., "Numerical simulation of Nusselt-Rayleigh correlation in Bénard cells. A solution based on the network simulation method," International Journal of Numerical Methods for Heat \& Fluid Flow, vol. 25, no 5, pp. 986-997, 2015.

[6] C. F. González-Fernández, Applications of the network simulation method to transport processes, in Network Simulation Method. Ed. J. Horno, Research Signpost, Trivandrum, India, 2002.

[7] Ngspice - Open Source mixed mode, mixed level circuit simulator (based on Berkeley's Spice3f5), 2016. [Online]. Available: http://ngspice.sourceforge.net/

[8] F. Alhama, Solución de problemas lineales y no lineales de transferencia de calor por el método de simulación por redes. Doctoral dissertation. Universidad de Murcia, 1999. 